\title{
Appetitive and aversive visual learning in freely moving Drosophila
}

\author{
Christopher Schnaitmann ${ }^{1,2}$, Katrin Vogt ${ }^{1,2}$, Tilman Triphan $^{2}$ and Hiromu Tanimoto ${ }^{1,2 *}$ \\ Max-Planck-Institut für Neurobiologie, Martinsried, Germany \\ Lehrstuhl für Genetik und Neurobiologie, Universität Würzburg, Würzburg, Germany
}

Edited by:

Martin Giurfa, Université Paul SabatierToulouse III, France

\section{Reviewed by:}

Makoto Mizunami, Hokkaido

University, Japan

Edith Roussel, Université Paul Sabatier,

France

\section{*Correspondence:}

Hiromu Tanimoto, Max-Planck-Institut

für Neurobiologie, Am Klopferspitz 18,

D-82152 Martinsried, Germany.

e-mail: hiromut@neuro.mpg.de
To compare appetitive and aversive visual memories of the fruit fly Drosophila melanogaster, we developed a new paradigm for classical conditioning. Adult flies are trained en masse to differentially associate one of two visual conditioned stimuli (CS) (blue and green light as CS) with an appetitive or aversive chemical substance (unconditioned stimulus or US). In a test phase, flies are given a choice between the paired and the unpaired visual stimuli. Associative memory is measured based on altered visual preference in the test. If a group of flies has, for example, received a sugar reward with green light in the training, they show a significantly higher preference for the green stimulus during the test than another group of flies having received the same reward with blue light. We demonstrate critical parameters for the formation of visual appetitive memory, such as training repetition, order of reinforcement, starvation, and individual conditioning. Furthermore, we show that formic acid can act as an aversive chemical reinforcer, yielding weak, yet significant, aversive memory. These results provide a basis for future investigations into the cellular and molecular mechanisms underlying visual memory and perception in Drosophila.

Keywords: behavioral assay, classical conditioning, chemical reinforcement, vision, method

\section{INTRODUCTION}

The fruit fly Drosophila melanogaster is an excellent model to study the genetic and neural bases of associative memory (McGuire et al., 2005; Pitman et al., 2009). Visual memories of the fly have been intensely studied using various aversive learning assays (Quinn et al., 1974; Spatz et al., 1974; Menne and Spatz, 1977; Lepot and Médioni, 1986; Wolf and Heisenberg, 1991; Le Bourg and Buecher, 2002; van Swinderen et al., 2009). In contrast to the relative abundance of aversive learning assays, only two behavioral paradigms for appetitive visual learning in Drosophila have been reported to date (Heisenberg, 1989; Gerber et al., 2004; see Fukushi, 1976, 1985, 1989 for studies on other fly species). However, appetitive and aversive visual memories have never been compared in the same setup. This is partially due to the limited compatibility of reinforcement application (i.e., exchangeable reward or punishment).

Few conditioning paradigms in insects are versatile enough to succeed in the direct comparison of mechanisms underlying appetitive and aversive memories. For example, in olfactory learning of Drosophila, paired presentation of an odor with sugar reward or electric shock punishment under otherwise same experimental conditions leads to approach or avoidance of the odor, respectively (Tempel et al., 1983; Schwaerzel et al., 2003; Gerber and Hendel, 2006; Honjo and Furukubo-Tokunaga, 2009). These opposite memories differentially recruit the two biogenic amines octopamine and dopamine, which respectively mediate appetitive and aversive reinforcements (Schwaerzel et al., 2003; Honjo and Furukubo-Tokunaga, 2009, but see Kim et al., 2007b; Selcho et al., 2009). Also in the olfactory conditioning of the sting extension reflex of the honey bee (Vergoz et al., 2007; Giurfa et al., 2009;
Roussel et al., 2009) and in visual and olfactory learning in the cricket (Unoki et al., 2005, 2006; Mizunami et al., 2009; Nakatani et al., 2009), appetitive and aversive memories can be directly compared in the same setup. Such comparisons yielded similar results as in Drosophila: appetitive and aversive reinforcement is mediated by octopamine and dopamine, respectively.

In order to understand and compare the mechanisms underlying visual appetitive and aversive memories in Drosophila, we sought to establish a new behavioral paradigm for visual associative learning in adult flies. This assay should: (1) produce reproducible associative memory, (2) be simple to set up and maintain, and (3) accommodate the application of different stimuli. We developed a classical conditioning protocol using a setup in which various visual and chemical stimuli can be simultaneously presented. We utilized an LCD screen to generate spectrally different visual stimuli that illuminate flies in a cylindrical arena. Chemical stimuli were presented on the bottom of the arena. We analyzed the effect of critical parameters for the formation of memories such as training repetition, order of reinforcement, interval between conditioned stimuli (CSs), motivation, and the impact of appetitive and aversive reinforcers on visual memory formation.

\section{MATERIALS AND METHODS FLIES AND PREPARATION}

The wild type Drosophila melanogaster strain Canton S was employed throughout the study. Flies were reared on standard cornmeal medium at $25^{\circ} \mathrm{C}$ and $60 \%$ relative humidity under a 14-h light/10-h dark cycle. All flies were handled without anesthesia until experiments and used 2-6 days after eclosion. 
Before starvation, flies were collected within 1 day after eclosion and kept in new food vials for at least 1 day to control their feeding status. At the beginning of experiments, they were transferred to moistened empty vials and starved for either $24-28$ or $48-52 \mathrm{~h}$, roughly calibrated by mortality (see Section "Results" for further details). Water was provided by means of tissue paper on the vial bottom and folded filter paper $(\varnothing 90 \mathrm{~mm})$ clamped by a slit of a plug.

\section{APPARATUS}

Flies in a Petri dish arena were illuminated from below through filter paper that contained a chemical substance. The experimental setup consisted of three major parts: (1) an LCD monitor used to generate visual stimuli; (2) a cylindrical arena, where flies were trained and tested; (3) a video device that recorded the back-lit arena (Figure 1).

The LCD monitor (MM19SE, ASUS Computer GmbH, Ratingen, Germany) was horizontally laid and was used to generate visual stimuli that were fitted to the Petri dish diameter (Figure 1). For conditioning, green $(0: 255: 0$ in R:G:B) or blue (0:0:255) stimuli were presented on a black background (0:0:0). For preference analyses, the arena was illuminated with red light (200:0:0). Figure 2 shows the spectral and intensity characteristics of these stimuli.
Filter paper soaked with sucrose (dried), water or acid solution increased light transmission by 40 or $100 \%$, respectively. Scheduled Slide Show function in PowerPoint 2007 (Microsoft Corporation, Redmond, WA, USA) was used to automatically change the visual stimuli. Routinely, four Petri dishes were placed on the monitor in fixed positions using a plastic frame (Figure 1B).

The cylindrical arena consisted of a Petri dish $(\varnothing 92 \mathrm{~mm}$, Sarstedt, Nümbrecht, Germany) on which flies could freely move, a pipe wall, and a second Petri dish used for a lid (Figure 1A). A circular piece of filter paper [round filter, $\varnothing 90 \mathrm{~mm}$ (trimmed to $84 \mathrm{~mm}$ when used), Hartenstein, Würzburg, Germany) was put on the floor of the Petri dish, depolarizing the light generated by the LCD screen (Figure 1A). Chemical substances were applied with the filter paper. A plastic ring that fitted to the inner diameter of a Petri dish (outer $\varnothing$ $84 \mathrm{~mm}$, inner $\varnothing 79 \mathrm{~mm}$, height $5 \mathrm{~mm}$ ) was used to clamp the filter paper to the dish and to fix the cylindrical pipe (Figure 1A).

The pipe had a black surface $(\varnothing 79 \mathrm{~mm}$, height $139 \mathrm{~mm})$ and served both as a wall to prevent flies from flying away and as a space to keep flies when exchanging Petri dish arenas (Figure 1A). Its smooth inner surface was coated with Fluon (Fluon ${ }^{\circledR}$ GP1, Whitford Plastics Ltd., UK) to prevent flies from hanging on the wall. Consequently, flies were forced to stay on the filter paper at the bottom of the arena (Figure 1C).

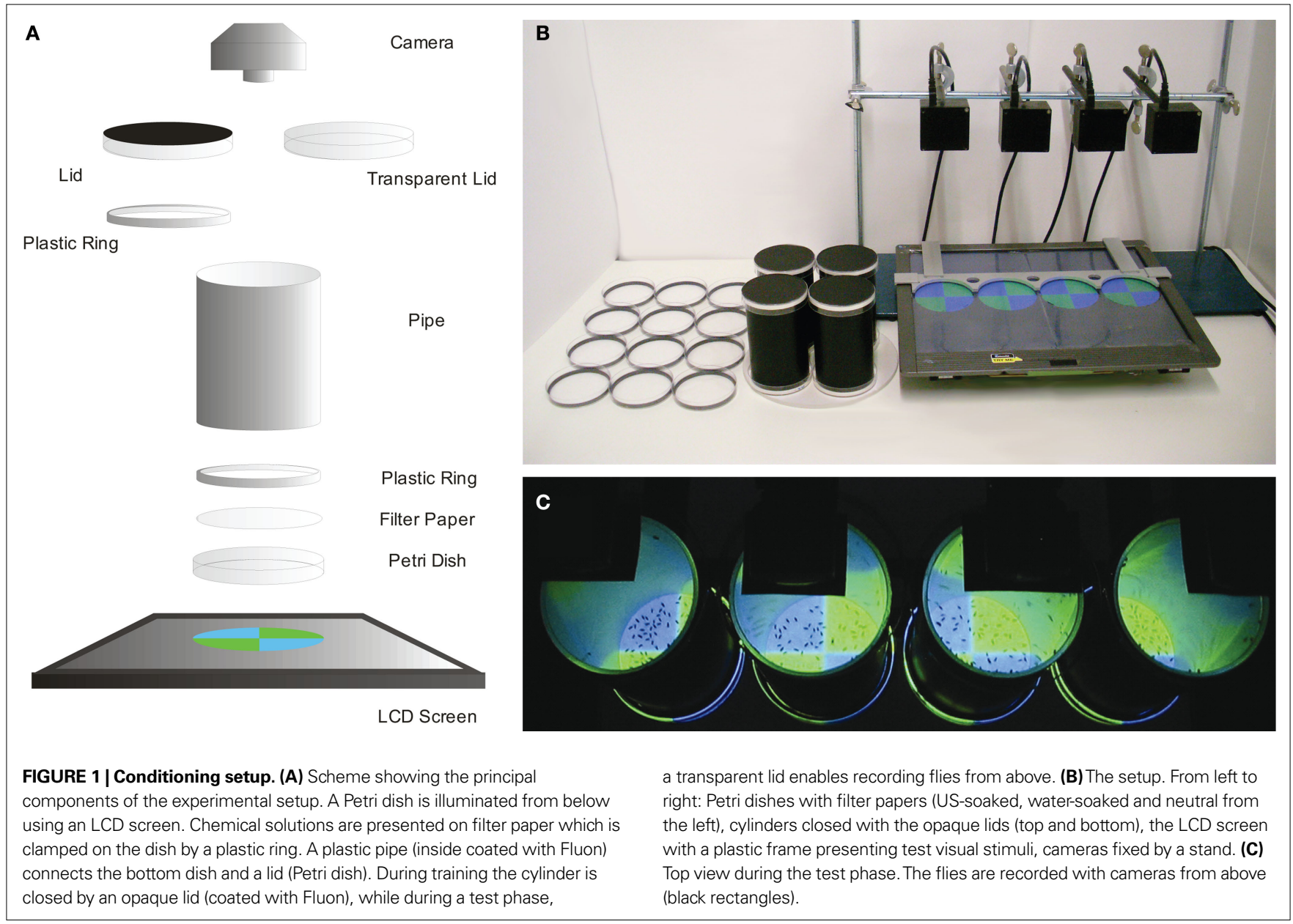




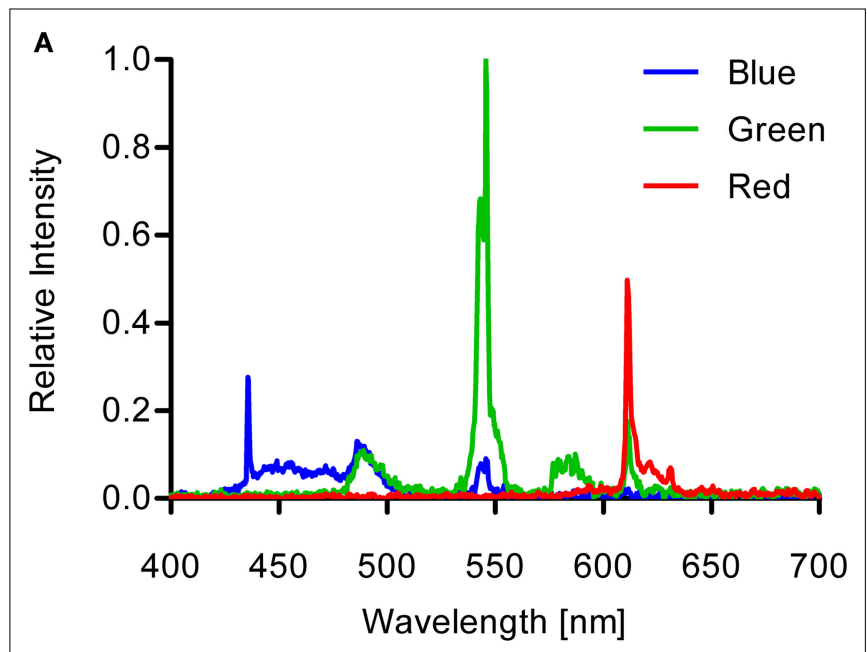

B

\begin{tabular}{|c|c|}
\hline Stimulus & Intensity [cd m-2] \\
\hline Blue & 3.1 \\
\hline Green & 24.5 \\
\hline Red & 5.0 \\
\hline
\end{tabular}

FIGURE 2 |Visual stimulus properties. (A) Spectra of blue, green and red stimuli generated by the LCD screen. (B) Intensities of the different light stimuli.

A Petri dish, transparent or opaque (laminated with black foil), was used for a lid (Figure 1A) at the top. During training, we used the opaque lid coated with Fluon and containing a plastic ring (see above). During the test phase, we used the transparent Petri dish (Figure 1A).

During the test phase, the arena (Petri dish floor) was video recorded from above with a CMOS camera (Sansun Webcam SN-509A, SANSUN, Deutschland, Germany; Easy cam, Typhoon, Germany, or Firefly MV, Point Grey, Richmond, Canada) (Figure 1C). We developed software that allows setting of four cameras independently (e.g., brightness, exposure time, frame rate, recording delay, compression, etc.). Typically, each arena was recorded for $90 \mathrm{~s}$ of the test at one frame per second.

\section{CONDITIONING}

\section{Conditioned and unconditioned stimuli}

All experiments were performed in darkness. During the training phase, the whole arena was illuminated from the bottom either with green or blue light, which were used as CS in a differential conditioning procedure (CS; Figure 3A; see Figure 2 for visual stimulus properties). In the test phase, the four quadrants of the arena were separately illuminated with green or blue light so that flies had to choose between both stimuli. Diagonal quadrants were illuminated with the same stimulus (Figures 1C and 4A).

The spectra of the visual stimuli generated by the subpixels of the LCD monitor were measured with a CCD spectrometer (Tristan USB, m-u-t AG, Wedel, Germany). A Luminance meter (BM-9, TOPCON TECHNOHOUSE CORPORATION, Tokyo, Japan) was used to measure the intensities of the stimuli (Figure 2).
Filter paper was used to present the unconditioned stimuli (US). In appetitive conditioning, sucrose was used as a US as it proved to be the most efficient reward among five different sugars in olfactory learning (data not shown). The filter paper was soaked with 1.5 M sucrose solution and subsequently dried. Filter paper that was presented with the non-reinforced stimulus was soaked with water and subsequently dried. In the test phase following appetitive conditioning, no US was presented; only visual stimuli and untreated filter papers were presented.

In aversive conditioning, either $1 \mathrm{M}$ acetic acid or $1 \mathrm{M}$ formic acid solution was used as a US. Contrarily to appetitive conditioning, the filter paper on which these solutions were applied was not dried. Accordingly, the filter paper that was presented with the non-reinforced light was soaked with the same amount of water. The test arena contained either a dry filter paper previously soaked with water or a filter paper soaked with the same acid solution as the US (see Section "Results" for further details).

\section{Appetitive conditioning}

A group of flies was trained following a differential conditioning procedure, i.e., only one of two consecutively presented visual stimuli (i.e., green and blue light) was paired with a sucrose reward (Figure 3). Two groups of flies were reciprocally trained regarding CS-US contiguity: Green+/Blue- and Blue+/Green- (Figure 3A). Typically, four groups of animals were simultaneously trained and tested in parallel (Figures 1B,C).

Using an aspirator, 50-100 flies were introduced into the cylinder from the lid. A switch of US presentation was carried out by exchanging Petri dishes at the bottom. First, the cylinder was inverted and after a gentle tap, the Petri dish now being on the top was quickly replaced by a new Petri dish (with or without US). Subsequently, flies were transferred to the new dish by inverting the cylinder again and by delivering a further gentle tap. This "inversion \& tap" procedure was done using a soft mouse pad and intended to transfer the flies by detaching them from a Petri dish. It cannot therefore be assimilated with the aversive shaking used for reinforcement in other conditioning protocols of Drosophila (Menne and Spatz, 1977; Mery and Kawecki, 2005; van Swinderen et al., 2009). Finally, the cylinder was immediately put in a fixed position on the LCD screen (Figure 1B).

Training (first CS presentation) started approximately $60 \mathrm{~s}$ after the introduction of the flies. The duration of CS/US presentation was $60 \mathrm{~s}$ (Figure 3B). An inter-CS interval (ICSI) caused by a fly transfer lasted typically 10-12 s (Figure 3B). In the experiment with a longer ICSI, the bottom dish was removed and replaced with a Fluon-coated lid, and flies were kept in this double-lid arena during the interval. Prior to the next CS, one of the lids was replaced with another Petri dish. Such a training trial was repeated four times unless otherwise stated.

After the last training trial, flies were placed on a dish without US. During the test phase, a transparent lid was used for video recording from top (Figure 1A). The test of immediate memory started $\sim 60 \mathrm{~s}$ after the offset of the last CS (Figure 3B), and the preference of trained flies for the two visual stimuli was recorded for $90 \mathrm{~s}$ (Figure 1C). For testing longer retention performances (Figure 8), flies were transferred into moistened empty vials using 


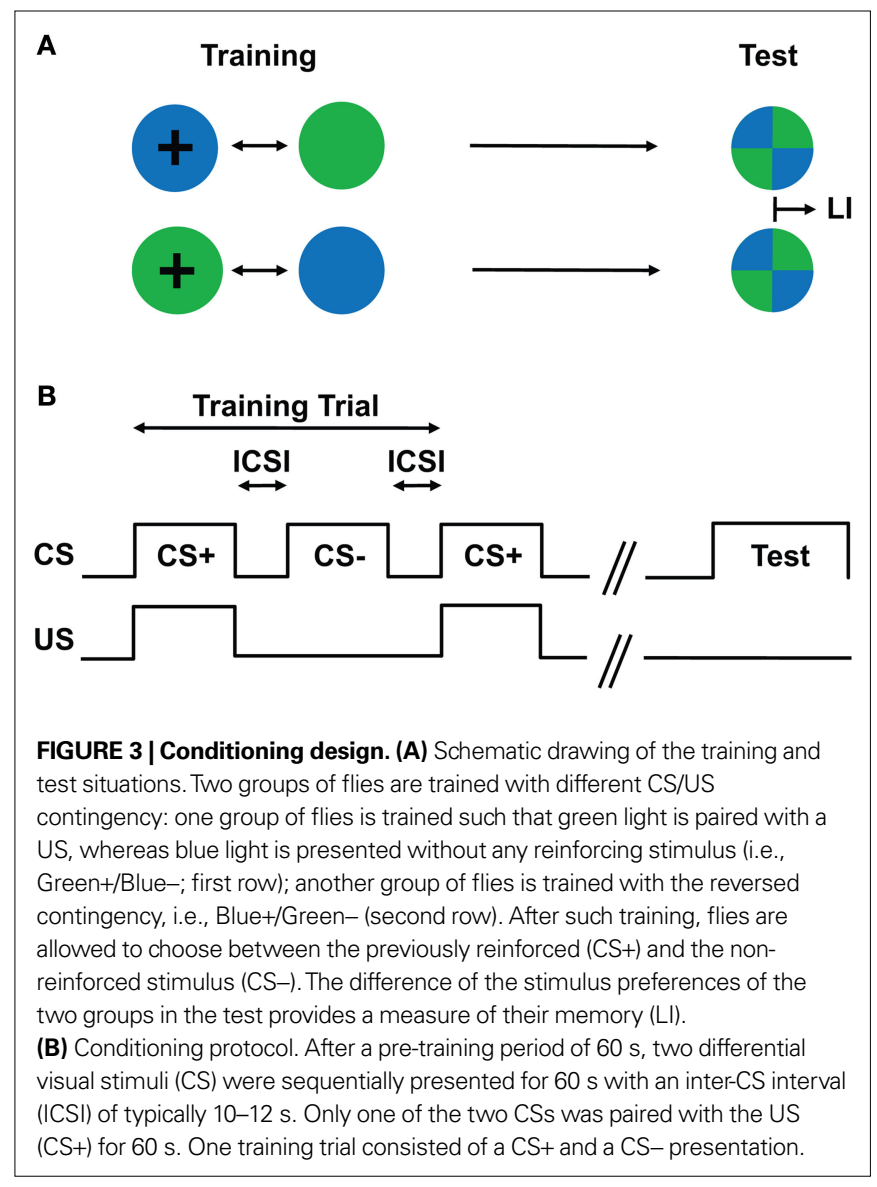

a funnel after training. The flies were kept in darkness until the test. The test started approximately $60 \mathrm{~s}$ after reintroduction of the flies.

The reciprocal experiment was performed immediately afterwards using the identical apparatus. In the reciprocal experiment, the non-reinforced stimulus of the first experiment was now paired with the US; i.e., if the green light was rewarded in the first experiment (Green+/Blue-), the reciprocal group experienced blue with the reward (Blue+/Green-; Figure 3A). The difference of these reciprocally trained groups in the visual stimulus preference was used to calculate a learning index (LI; see below).

To exclude a non-associative effect that stems from the order of reinforcement, approximately one half of the experimental groups received the US together with the first visual stimulus (CS+/CS-), and the other half received the US with the second stimulus (CS-/CS+).

\section{Aversive conditioning}

The training protocol was identical to that of appetitive conditioning with the difference that aversive chemical substances were used as US. The interval between the training and the test phases was $90 \mathrm{~s}$, during which the cylinder was closed by lids at the bottom and top. Shortly before the test phase started, one lid was replaced with a Petri dish for a respective test (see Section "Results" for details), and the other was replaced with a transparent lid. Trained flies were tested both in the presence or absence of the US.

\section{STIMULUS PREFERENCE}

To test the attractive/aversive nature of a chemical substance, the flies' preference was also measured using the cylindrical arena. To provide a choice situation, a piece of circular filter paper was cut into two halves. These halves were laid next to each other in a Petri dish and clamped with a plastic ring (Figure 1A). Each half presented water or the respective test substance. Papers were freshly prepared prior to an experiment to avoid desiccation. The arena was back-lit in red for video recording (see Figure 2 for visual stimulus properties). Except for these adaptations, the setup was identical to that used for conditioning.

Approximately 50 flies were directly introduced into the choice arena after recording was started. The arena was recorded for $240 \mathrm{~s}$. To avoid potential positional biases, the sides of the test substance and the control were alternated.

\section{VIDEO RECORDING AND DATA ANALYSIS}

Stimulus preference was determined by the flies' distribution. To this end, we counted in every frame of our video recordings the number of flies in each region of interest of the arena (i.e., diagonal quadrants in the case of conditioning experiments and a half arena in the case of preference experiments). All videos in this study were recorded at one frame per second.

The number of flies was scored semi-automatically using a preset macro for Image (Rasband, W.S., ImageJ, U. S. National Institutes of Health, Bethesda, MD, USA; Figure 4). First, the video was grayscaled, the circular arena in the video frames was cropped, and the surrounding was cleared (Figure 4A). Each region of interest (see above) was outlined (Figure 4A) and flies located therein were counted using the "Analyze Particles" function after manually setting a threshold that separated the flies from the background and a size range that excluded non-fly particles (Figures 4A,B). The threshold was determined by eye while the size range was set according to a histogram of the particle area in the respective quadrant (Figure 4B). Flies touching a border of two compartments were excluded. The error rate of this automated counting procedure was calculated by comparing manual and machine based counting in 20 randomly selected quadrants. It ranged from two to nine percent depending on the camera used.

\section{PREFERENCE AND LEARNING INDICES}

Conditioned behavior was quantified based on the flies' preference for the CS in both reciprocal experiments (Tully and Quinn, 1985; Rescorla, 1988; Scherer et al., 2003). We first calculated a preference index for green $\left(\mathrm{PI}_{\mathrm{G}}\right)$ for each time point as the number of flies on the Green quadrants (\#Green) minus the number on the blue quadrants (\#Blue) divided by the total number of flies counted. $\mathrm{PI}_{\mathrm{G}}$ was calculated in both reciprocal experiments [i.e., Green+/Blue$(\mathrm{G}+\mathrm{B}-)$ and Blue+/Green- $(\mathrm{G}-\mathrm{B}+)]$ :

$$
\mathrm{PI}_{\mathrm{G}}(\mathrm{G}+\mathrm{B}-)=(\# \mathrm{Green}-\# \mathrm{Blue}) / \# \text { Total }
$$

$$
\mathrm{PI}_{\mathrm{G}}(\mathrm{G}-\mathrm{B}+)=(\# \text { Green }- \text { \#Blue }) / \# \text { Total }
$$

$\mathrm{PI}_{\mathrm{G}}$ values can thus range from -1 to 1 . Positive values indicate that more flies prefer the "green" quadrants whereas negative values indicate that more flies prefer the "blue" quadrants. To quantify the flies' preference/avoidance for a chemical substance, a PI was calculated and analyzed in the same way: the difference of the number of flies in the two halves was divided by the total number of flies. 


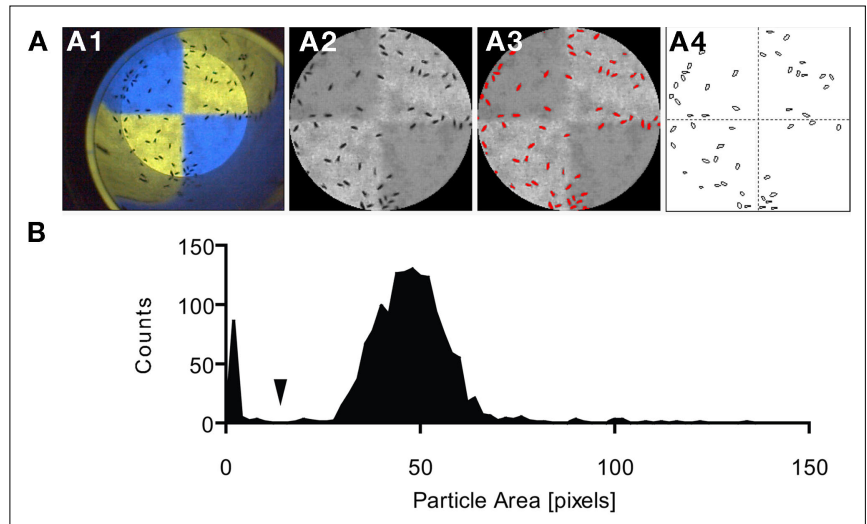

FIGURE 4 | Semi-automatic fly counting. Using ImageJ, flies in every region of interest (e.g., quadrant) were counted in every frame (automated using a macro). (A) The raw image (A1) was trimmed by manually selecting the test arena, and the image was gray-scaled (A2). A threshold was set to separate the flies from the background (A3; recognized objects marked in red). The "Analyze Particles" function was used to count the objects in each quadrant and frame (A4). (B) Histogram of the particle area distribution on a quadrant of one test video. Particles smaller than a certain size (arrow) were regarded as non-fly particles and excluded from counting.

For single-fly conditioning, the difference between the number of frames where a given fly was on the Green and Blue quadrants was taken as a preference.

An LI was calculated by subtracting $\mathrm{PI}_{\mathrm{G}}$ values of the two reciprocally trained groups and by dividing the resulting value by 2 :

$\mathrm{LI}=\left[\mathrm{PI}_{\mathrm{G}}(\mathrm{G}+\mathrm{B}-)-\mathrm{PI}_{\mathrm{G}}(\mathrm{G}-\mathrm{B}+)\right] / 2$

Like a preference index, $\mathrm{LI}$ can range from -1 to 1 . A positive LI indicates conditioned approach, whereas a negative LI indicates conditioned avoidance. If flies do not show associative memory in the test, the LI would become 0 .

The LI was calculated in each frame of a recorded video. For bar graph presentation and statistical comparisons of different groups, all single LIs in the entire test phase (1-90 s) were averaged.

\section{STATISTICS}

The significance level of all statistical tests was set to 5\%.All groups were first tested for normal distribution with the Shapiro-Wilk test with Bonferroni correction and for homogeneity of variance with Bartlett's test or F-test. As in no case our data significantly violated the assumption of the normal distribution, parametric comparisons [i.e., one-sample $t$-test with Bonferroni correction, Student's and Welch's $t$-tests, one-way ANOVA followed by planned pair-wise comparisons (Bonferroni)] were applied as specified in the figure legends. All statistical calculations were performed using the software Prism 5 (GraphPad, San Diego, CA, USA).

\section{RESULTS}

\section{APPETITIVE VISUAL LEARNING}

\section{Repetition of training and order of reinforcement}

First, we examined the effect of training repetition. Twenty-four hour starved flies were subjected to $0,1,2,4$ or 8 cycles of a training trial. For the group with no training trial, flies were randomly assigned to the groups "green rewarded" or "blue rewarded."
In the test phase, flies having undergone four trials of a sucrose reward in the presence of green light showed a higher preference for the green stimulus than the preference for blue exhibited by the reciprocal group rewarded on blue light (Figure 5A). Flies without training (naïve flies) showed a significant preference for the green light $(P<0.001)$. This might explain the shift of preference toward green after training (Figure 5A). By analyzing the time course of memory performance (i.e., differences in the preferences), we found that the choice of all trained groups reached an asymptote within $20 \mathrm{~s}$ (Figure 5B).

All the trained groups showed a significant conditioned approach to the rewarded stimulus $(P<0.01)$ while no significant associative memory was detected in the group without training $(P>0.05$; Figures $\mathbf{5 B}, \mathbf{C})$. The performance of the trained groups was significantly better than that of the group without training $(P<0.01$; Figure 5C). Comparison among the groups with training (one to eight trials) revealed a significant difference $(P<0.05)$. The memory tended to increase with training repetition $(P<0.05$; comparison of groups trained with two and four trials; Figure 5C).

In order to analyze potential non-associative effects of reinforcement order (Tully and Quinn, 1985; Kim et al., 2007a), we discriminated LIs according to whether sugar was delivered with the first or second CS in each trial (i.e., CS+/CS- vs. CS-/CS+, respectively; Figure 5D). We compared the performance of these two groups and found that the order of reinforcement did not significantly affect memory $(P>0.05$, Figure 5D for the groups with four-cycle training trial; data not shown for the groups with one-, two-, and eight-cycle trials). As the intervals between the last US presentation and the test are different in these two groups, the periodical stimulus presentation protocol did not significantly modulate visual memory. Since such a non-associative effect might however become evident only in some mutants or under certain experimental conditions (Acevedo et al., 2007), we randomized the order of reinforcement in all groups of this study. In the following experiments four-cycle training was used since immediate memory reached an asymptote (Figure 5C).

\section{Different inter-CS intervals}

In our associative training, we alternately presented two color stimuli. To examine the role of the interval between the two colored cues, we trained flies with different ICSIs (10,30 or 90 s, Figure 6A). All other training and test conditions were kept constant, while the total length of each training protocol differed accordingly. All groups displayed significant conditioned behavior (Figure 6A) and their performances did not significantly differ from each other $(P>0.05)$. Therefore, the ICSI of $10 \mathrm{~s}$ was adopted for further experiments.

\section{Starvation period}

Appetitive learning requires appropriate appetitive motivation, which can be varied through starvation of flies (Tempel et al., 1983; Krashes and Waddell, 2008; Colomb et al., 2009). The resistance to starvation is controlled by many physiological factors and appears to fluctuate significantly. All results described above were obtained from flies starved for $24 \mathrm{~h}$. This starvation period yielded a level of mortality around $20 \%$. In order to measure the effect of the starvation period on visual learning, three groups of flies were starved for 

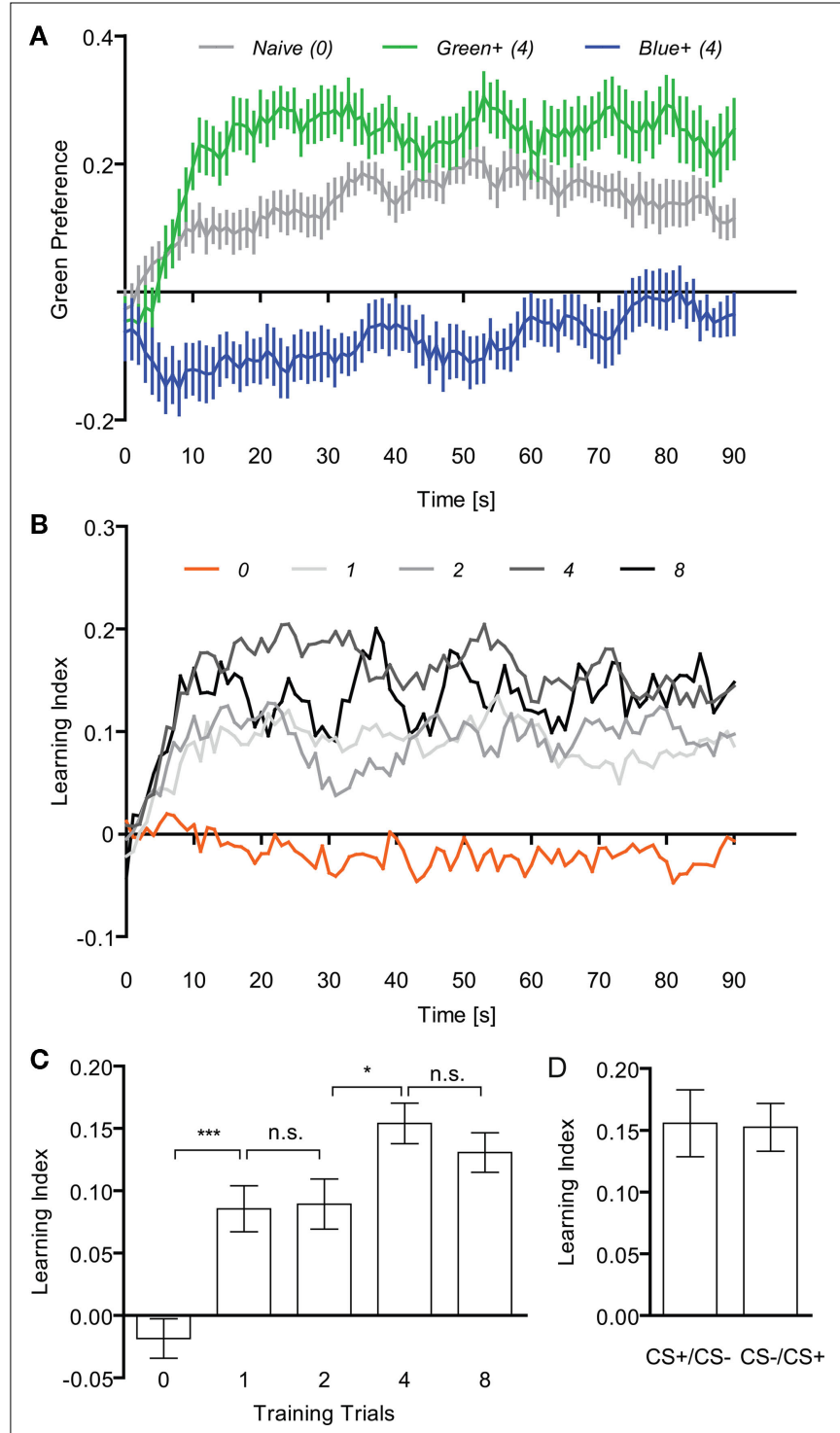

FIGURE $\mathbf{5}$ |Appetitive visual associative memory. (A) Time course of the mean preference for the green stimulus of naive flies (no training) and flies that received four training trials [two reciprocal groups: Green+/Blue- (green) and Blue+/Green- (blue). Flies that received a sugar reward with green light showed a higher preference for the green stimulus during the test phase than the reciprocal group having received the same reward with blue light. (B) Time course of the mean LIs of flies with $(1,2,4$ or 8$)$ or without (0) training trials. (C) Effect of training repetition. Without training no significant memory could be found [one-sample $t$-test, $\left.t_{\{13)}=1.159, P>0.05\right]$, whereas all trained groups showed significant memory [one-sample t-test, one trial: $t_{(17)}=4.632, P<0.01$; two trials: $t_{(19)}=4.446, P<0.01$; four trials: $t_{(19)}=9.490, P<0.001$; eight trials: $\left.t_{(17)}=8.242, P<0.001\right]$. Comparison among the groups with training (one to eight trials) revealed significant difference [one-way ANOVA, $F_{(3,72)}=3.512$, $P<0.05]$. The memory tended to increase with training repetition [e.g., comparison of groups trained with two and four trials: $t$-test, $t_{138 \mid}=2.632$, $P<0.05] . n=18-20$. (D) Effect of the order of CS presentation (four trials). The same data as in C were sorted to CS+/CS- and CS-/CS+ and reanalyzed. Either the first or second visual stimulus of each training trial was paired with the US. No significant difference was found between both groups [t-test, $t_{\text {18. }}=0.096$, $P>0.05, n=10]$. In all the diagrams, bars (points) and error bars indicate means and the standard error of the mean, respectively. Asterisks indicate statistical significance $\left({ }^{*} P<0.05 ;{ }^{*} P<0.01 ;{ }^{* *} P<0.001\right)$.
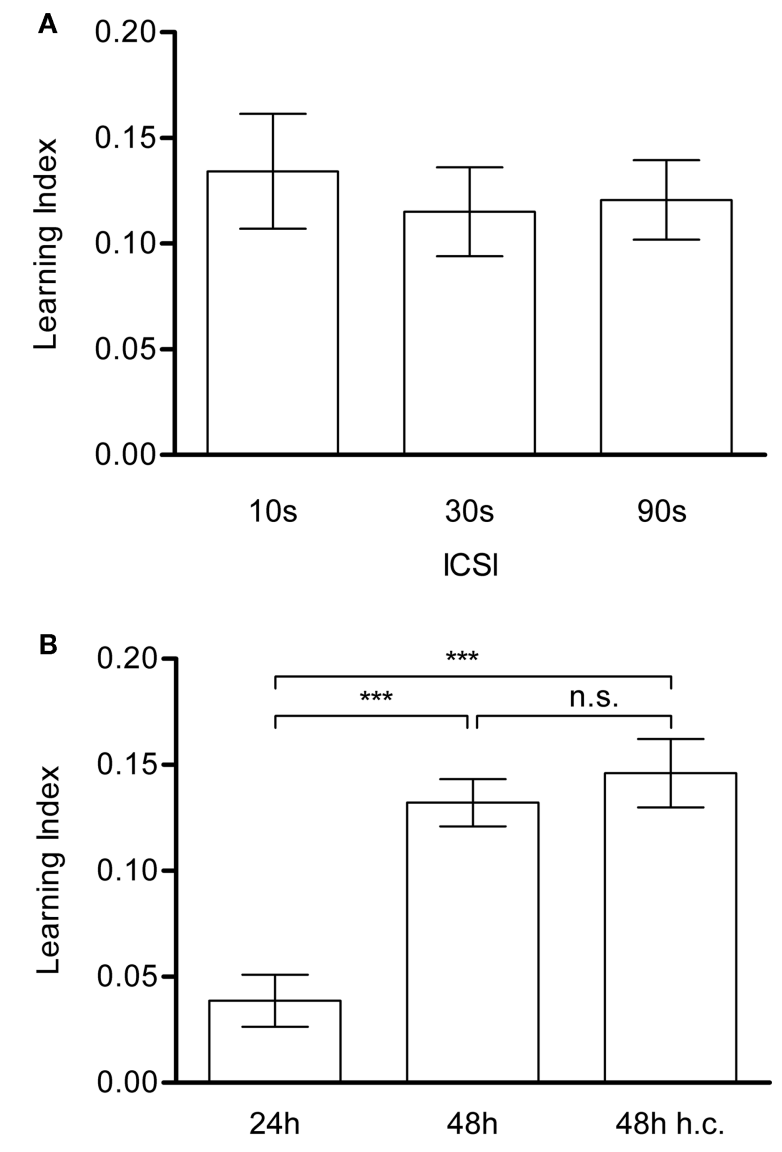

Starvation Period

FIGURE 6 | Inter-CS interval and starvation-dependency of visual appetitive memory. (A) The effect of duration of the inter-CS interval (ICSI). The interval lasted 10,30 or $90 \mathrm{~s}$. All groups showed significant memory [onesample $t$-test, $10 \mathrm{~s}: t_{(19)}=4.925, P<0.001 ; 30 \mathrm{~s}: t_{(19)}=5.476, P<0.001 ; 90 \mathrm{~s}:$ $\left.t_{\text {t171 }}=6.394, P<0.001\right]$ while no significant difference could be found among all groups [one-way ANOVA, $F_{(2.55)}=0.1897, P>0.05$ ]. $n=18-20$. (B) The effect of starvation periods. Flies were starved for 24,48 or $48 \mathrm{~h}$ at high humidity conditions (h.c.). All groups showed significant memory lone-sample t-test, 24 h: $t_{t 17)}=3.154, P<0.05 ; 48$ h: $t_{t 15)}=11.87, P<0.001 ; 48$ h h.c.: $\left.t_{(12)}=9.022, P<0.001\right]$, while longer starvation resulted in a significantly higher performance than short starvation [t-test, $\left.t_{321}=5.288, P<0.001\right]$. Different humidity conditions had no significant effect on the conditioned approach [t-test, $\left.t_{[27]}=0.7242, P>0.05\right] . n=13-16$.

two different periods. The first group was starved for $24 \mathrm{~h}$ as in the previous experiments. However, almost no dead flies were found in the starvation vials at the time point of experiments, suggesting that resistance to starvation may change over time. The second and third group were starved for $48 \mathrm{~h}$ and yielded a mortality rate that was similar to that of the previous experiments (see Figures 5 and $\mathbf{6}$ ). To prevent potential water deprivation caused by long starvation, the third group was kept in a humidified box.

Although all three groups acquired a significant memory $(P<0.05$; Figure 6B), the performance of the $24 \mathrm{~h}$-starved flies was significantly lower than that of the flies starved for $48 \mathrm{~h}(P<0.001$; Figure 6B). The high humidity during the starvation did not cause a significant difference $(P>0.05$; Figure 6B). Thus, these results indicate that the expression of visual appetitive memory varies 
with the amount of starvation, and that this variation is not due to water deprivation. As the performance of $48 \mathrm{~h}$-starved flies was comparable to that of flies in the previous experiments with 24 h-starvation (see Figures 5 and 6A), the calibration of a starvation period might be necessary to stably assess appetitive memory.

\section{Single-fly conditioning}

Typically, 50-100 flies were collectively trained and tested. However, this en masse conditioning might not reflect single fly behavior, as the choice of individual flies could be influenced by other flies (Chabaud et al., 2009). Therefore, we examined whether the performance of flies that were trained and tested in a group was different from that of flies that were trained and tested individually. We compared the LIs obtained after single-fly conditioning and after en masse conditioning. The former is based on the time spent on the CS+ and CS-, whereas the latter results from the differential distribution of flies. LIs of flies that underwent training and test in a group (Group) or individually (Individual) did not differ significantly from each other $(P>0.05)$ despite presenting significantly different variances $(P<0.001$; Figure 7$)$. This suggests that the choice of individual flies in our previous tests was not affected by the en masse protocol.

\section{Memory retention}

To address the stability of appetitive visual memories, flies were tested $5 \mathrm{~min}, 1,3$ or $6 \mathrm{~h}$ after receiving four training trials. After the training, flies were removed from the arena and kept in a vial during the respective retention interval. The memory decayed to ca. 50\% within 3 h (Figure 8). It diminished after 6 h (Figure 8).

\section{AVERSIVE VISUAL LEARNING \\ Aversive chemical substances}

To find a chemical that might function as an aversive reinforcer for visual conditioning, we examined the flies' avoidance when confronted with different substances. We chose three different acids,

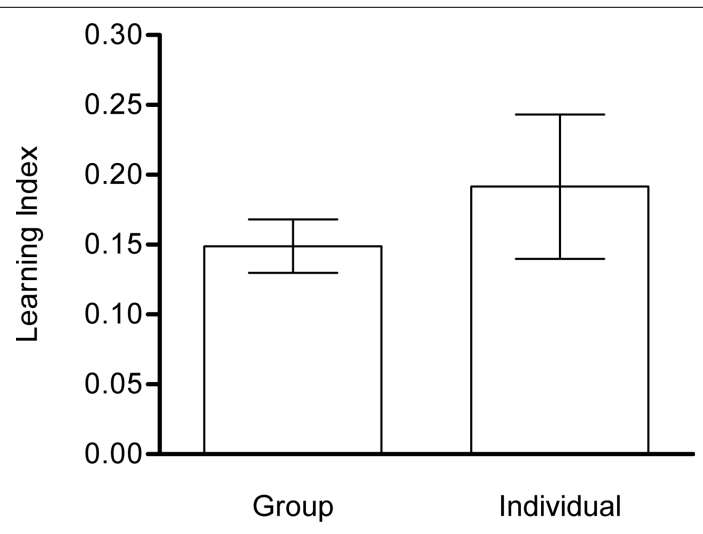

FIGURE 7 | Single-fly vs. en masse conditioning. Learning indices of flies that undergo training and test in a group (Group) or individually (Individual) did not differ significantly from each other [Welch's $t$-test, $t_{(27)}=0.7742, P>0.05$ ] despite significantly different variances $\left[F\right.$-test, $\left.F_{(22,16)}=0.859, P<0.001\right)$. Both groups showed significant memory [one-sample $t$-test, Group: $t_{(16)}=7.802$, $P<0.001$; Individual: $t_{(22)}=3.716, P<0.01$ ]. Note that the learning index of single-fly conditioning is based on the time spent on CS+ and CS-, whereas memory of en masse conditioning is measured with the differential distribution of flies. $n=17$ and 23 . one inorganic (phosphoric acid) and two organic ones (formic acid and acetic acid). Acetic acid was previously shown to be aversive to flies (Joseph et al., 2009). Additionally, we measured avoidance of sodium chloride and quinine as they were previously shown to be potent aversive reinforcers in fly conditioning (Quinn et al., 1974; Gerber and Hendel, 2006).

For the measurement of avoidance, the apparatus used for appetitive conditioning was slightly modified. The filter paper on the arena ground was split in two halves to create a choice situation: one half contained the substance to be tested and the other half the solvent used to dilute the substance (i.e., water). As in the previous quantification of aversive memory, a preference index was determined based on the distribution of the flies on the two halves.

Both organic acids, formic acid and acetic acid, strongly repelled flies at a concentration of $1 \mathrm{M}(P<0.001$, Figure 9A). Lower concentrations barely evoked avoidance (Figure 9A). Interestingly, we found that phosphoric acid did not repel flies at any tested concentration although it was more acidic than acetic acid or formic acid at $1 \mathrm{M}(P>0.05$, Figure 9A). Similarly, sodium chloride and quinine (both at the maximum soluble concentrations; 6 and $0.1 \mathrm{M}$, respectively) did not evoke significant avoidance in our setup $(P>0.05$, Figure 9B). Thus, we used acetic acid and formic acid as potential aversive reinforcers in our conditioning experiments.

\section{Aversive visual learning}

To allow comparison between aversive and appetitive conditioning, we applied the same training protocol as for appetitive learning except for the use of different reinforcing substances. Flies were starved and trained with four conditioning trials in the same apparatus. No significant visual memory could be detected with $1 \mathrm{M}$ formic acid or $1 \mathrm{M}$ acetic acid when the test arena did not contain the corresponding acid $(P>0.05$, Figure 10). In Drosophila larvae, aversive olfactory memory can be expressed only in the presence of an aversive stimulus presented during the test situation (Gerber and Hendel, 2006). Thus, flies could have established an aversive visual memory but the absence of aversive reinforcer during the test might have prevented revealing such a memory. Therefore,

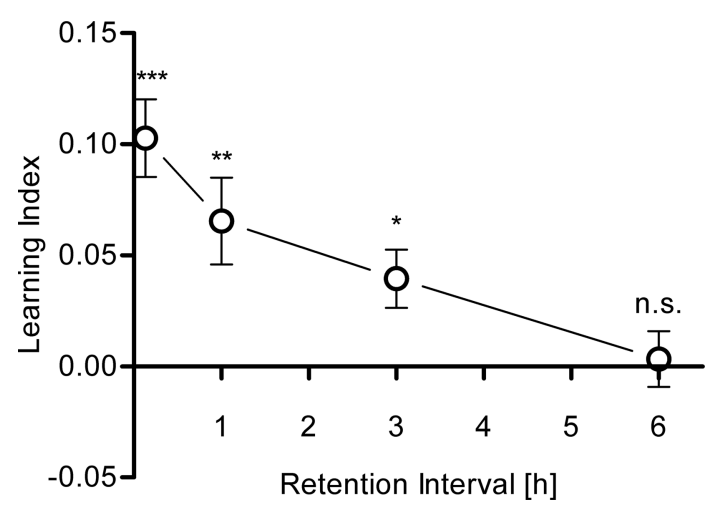

FIGURE 8 | Memory retention. Learning indices of flies that were trained with four training trials and tested after 5 min., 1, 3 and $6 \mathrm{~h}$. Significant memory was found up to $3 \mathrm{~h}$ after training [one-sample t-test, $5 \mathrm{~min}$ : $\left.t_{(34)}=5.891, P<0.001 ; 1 \mathrm{~h}: t_{(34)}=3.358, P<0.01 ; 3 \mathrm{~h}: t_{(29)}=3.008, P<0.05\right]$ After $6 \mathrm{~h}$, no significant memory was detected anymore [one-sample $t$-test, $\left.t_{(15)}=0.2622, P>0.05\right] . n=16-35$. 


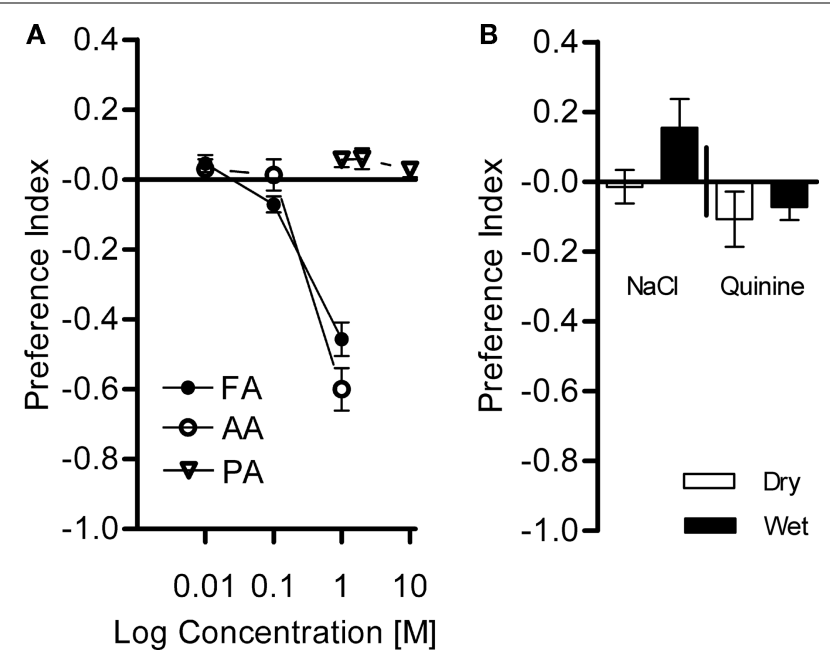

FIGURE 9 |Avoidance of diverse chemical substances. Choices between different chemical solutions and the control (water) were given to naïve flies. (A) Acid avoidance. Flies were tested with formic acid (FA), acetic acid (AA) at 0.01-1 M, and phosphoric acid (PA) at 1-10 M. Strong avoidance was found for FA and $\mathrm{AA}$ at $1 \mathrm{M}$ [one-sample $t$-test, $\mathrm{AA} 1 \mathrm{M}: t_{[7]}=16.73, P<0.001$; FA $\left.1 \mathrm{M}: t_{(7)}=15.13, P<0.001\right]$, whereas moderate avoidance, if at all, was observed at lower concentrations [one-sample $t$-test, $A A 0.01 \mathrm{M}: t_{i 7)}=1.134$, $P>0.05$; AA $0.1 \mathrm{M}: t_{(7)}=4.787, P<0.05 ;$ FA $0.01 \mathrm{M}: t_{(7)}=0.2669, P>0.05 ;$ FA $\left.0.1 \mathrm{M}: t_{[7]}=1.358, P>0.05\right]$. PA did not evoke a significant avoidance at any of the tested concentrations [one-sample $t$-test, PA $1 \mathrm{M}: t_{(7)}=1.356, P>0.05 ; \mathrm{PA}$ $2 \mathrm{M}: t_{(7)}=0.2009, P>0.05 ;$ PA $\left.10 \mathrm{M}: t_{(7)}=0.5641, P>0.05\right] . n=8$.

(B) Avoidance of $\mathrm{NaCl}(6 \mathrm{M})$ and quinine $(0.1 \mathrm{M})$. Both substances were assayed with dry or wet filter paper. No avoidance of the flies to these substances at any condition was found [one-sample $t$-test, $\mathrm{NaCl}$ dry: $t_{(9)}=0.3021, P>0.05 ; \mathrm{NaCl}$ wet: $t_{(9)}=1.872, P>0.05$; quinine dry: $t_{(9)}=1.353, P>0.05$; quinine wet: $\left.t_{(7)}=1.959, P>0.05\right] . n=10$.

we additionally measured the response of flies in a test situation in which the corresponding US was made available. Flies showed a small, yet significant, memory in the case of formic acid $(P<0.01)$, but not acetic acid (Figure 10). These results suggest that formic acid can act as an aversive reinforcer and that visual memories can be expressed if formic acid is presented in the test. Although acetic acid induced avoidance in a similar way as formic acid, it did not act as a reinforcer under the examined conditions.

\section{DISCUSSION}

\section{A NEW VERSATILE VISUAL CONDITIONING PARADIGM}

We successfully established a new behavioral paradigm for visual classical conditioning in adult Drosophila. Paired presentation of an appetitive or aversive chemical (US) and a visual stimulus (green or blue light; CS) significantly increased flies' preference/avoidance for the conditioned visual stimulus (Figures 5A and 10). All groups of flies were exposed to the same amount of CS and US; the only difference between two reciprocally trained groups was the CS-US contiguity. Thus, this conditioning design excludes the contribution of non-associative effects to the LI (Rescorla, 1988). We demonstrated that appetitive and aversive visual memories of the fly can be measured in the same setup (Figures 1, 5, and 10). This assay may therefore be applied to explore molecular and cellular mechanisms that dissociate appetitive and aversive visual

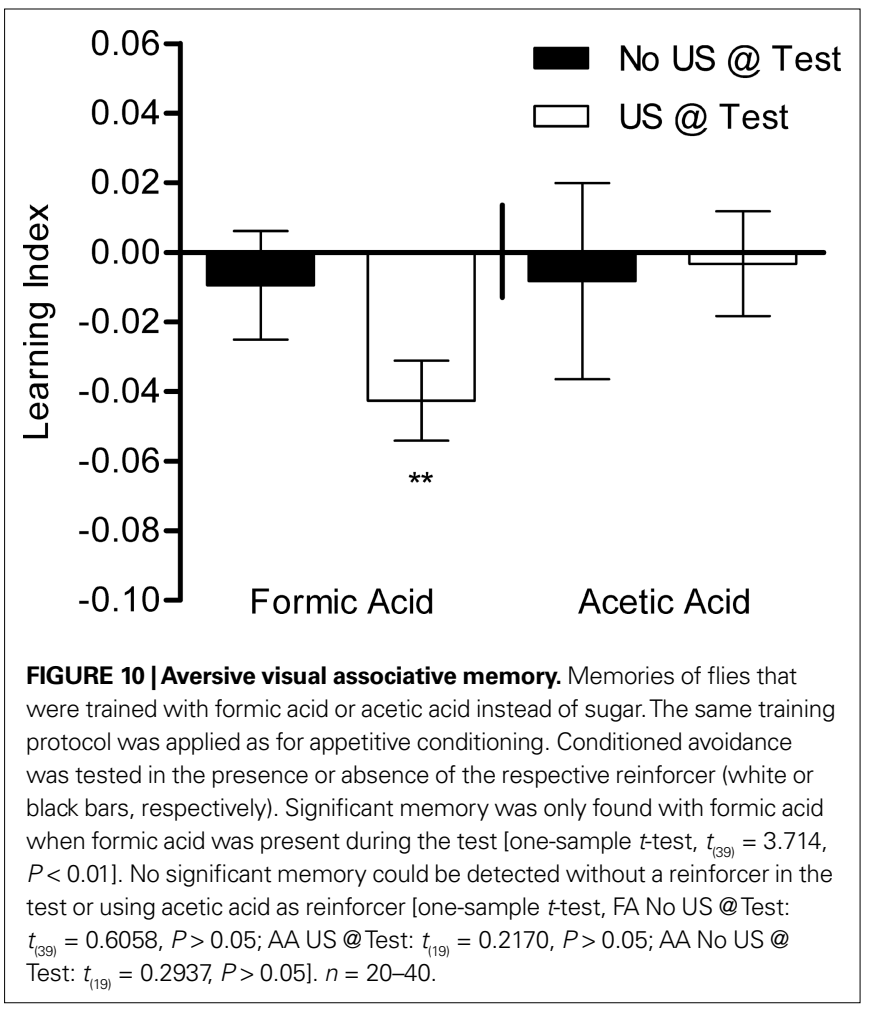

memories using Drosophila genetics. Such comparison has been previously done by combining a similarly versatile behavioral protocol and pharmacology in the cricket (Unoki et al., 2006; Nakatani et al., 2009). We also showed that memories of single-fly and en masse conditioning are not significantly different (Figure 7), so that we suppose that the behavior analyzed in the en masse assay reflects individual behavior, at least in the case of immediate appetitive memory (Figure 7). Collective behavior might become influential under some experimental condition, because a recent report on aversive olfactory conditioning revealed that a particular memory component is selectively affected in adult flies (Chabaud et al., 2009).

With a fixed time period of starvation, scores of appetitive memory seemed to fluctuate over a long time range (e.g., season). This fluctuation can be calibrated by varying the starvation period according to the mortality rate, implying that feeding motivation is correlated with resistance to starvation (Shiraiwa and Carlson, 2007) (Figure 6B). Thus, visual memory in our setup is independent from starvation resistance. This also suggests that a fixed time period of starvation may lead to different levels of feeding motivation, thus implying that starvation has to be calibrated in order to compare appetitive memories of different genotypes (Thum et al., 2007; Shiraiwa and Carlson, 2007).

Appetitive visual memories in our assay were retained for several hours (Figure 8). Although this performance is rather short compared to memory duration found in appetitive olfactory memory lasting more than $24 \mathrm{~h}$ (Krashes and Waddell, 2008; Colomb et al., 2009), it might still allow the analysis of different memory components (see Quinn and Dudai, 1976; Honjo and Furukubo-Tokunaga, 2009). Furthermore, by optimizing 
training conditions (e.g., application of spaced training or more repetitions), our protocol might be improved to allow the study of longer-lasting memories.

Furthermore, we demonstrated that this setup can be adapted to measure preferences for chemical and visual stimuli (Figure 9). It is also applicable to the analysis of many different kinds of behaviors that involve freely moving flies, such as circadian rhythm. Altogether, this new behavioral paradigm can become a significant alternative to hitherto established visual learning assays, as it is simple to set up and enables rapid and reproducible data acquisition.

In contrast to appetitive memory with sucrose, aversive memory was marginal with formic acid and not detectable with acetic acid, although they both induced similarly robust avoidance (Figures 9 and 10). Such differential processing of acetic acid in reflexive (unconditioned) and conditioned avoidance is similar to findings in Drosophila larvae (Schipanski et al., 2008). Aversive memory of formic acid was expressed only if it was presented during the test (Figure 10). This result corroborates conclusions and the rationale of a previous study using Drosophila larvae (Gerber and Hendel, 2006). Larvae only exhibited aversive memory if an aversive US was present in the memory test. It can be argued that in our case, the presentation of formic acid together with a previously not reinforced CS may induce a new aversive association that counteracts conditioned avoidance of the previous CS+. This is, however, an unlikely scenario in our experiments, because initial conditioned avoidance was maintained in the entire test period (90 s) without significant decrease (data not shown).

Since chemical stimulation of the tarsi or antennae was shown to signal less potent sugar reinforcement than the proboscis stimulation (Wright et al., 2007; de Brito Sanchez et al., 2008) and formic acid is unlikely to induce proboscis extension, weak aversive memory might be due to little contribution of the proboscis. Presentation of formic acid as an olfactory stimulus or testing flies with the defective olfactory system (e.g., Or83b ${ }^{-}$) may clarify the contribution of olfaction to formic acid perception. In any event, improvements of the protocol and/or US are therefore important for examining aversive memory in future.

\section{COMPARISON WITH OTHER VISUAL LEARNING ASSAYS}

Our assay has many similarities with the visual aversive learning paradigm developed by Menne and Spatz, where colored illumination of a vial was paired with vigorous shaking as aversive reinforcement (Menne and Spatz, 1977). Similar to our setup, the entire arena was illuminated, and freely moving flies were handled as a group (Menne and Spatz, 1977). Since they also applied differential conditioning and a discrimination task, this paradigm was successfully used in studies of color vision in Drosophila (Menne and Spatz, 1977; Bicker and Reichert, 1978; Hernández de Salomon and Spatz, 1983). Later, Gerber et al. (2004) established a behavioral paradigm for appetitive visual memory in larvae by illuminating an entire arena from the bottom. Encouraged by these successful precedents, we employed illumination of the entire arena for CS presentation (Figure 1). The application of a computer screen and a mechanism to keep flies on the bottom of the arena added versatility in CS and US presentation (Figure 1).
Visual learning in adult flies has been extensively studied using the so-called flight simulator (Wolf and Heisenberg, 1991). In this computer-controlled setup, a fruit fly flying stationary in the middle of a cylindrical arena learns that flying toward certain directions (i.e., quadrants) of the arena is permitted while flying toward other directions is punished by means of a heat beam. Different landmarks displayed on the wall of the arena signalize the safe and dangerous quadrants. Visual memory formed in the flight simulator is reproducibly robust (Heisenberg et al., 2001). In particular, presentation of complex visual objects as landmarks is possible (Liu et al., 2006). Our assay has two major differences compared to the flight simulator. First, the behavioral apparatus is much easier to set up and maintain, and involves less customized mechanics and electronics. Second, the demands for flies are less in our assay. In the flight simulator, the tethered fly needs to keep flying at least for several minutes in order to be trained and tested. Consequently, mutants with defective wings or flight cannot be examined in this apparatus (Brembs et al., 2007). Since the threshold of walking appears to be lower than that of flight in Drosophila, a broader range of mutants may be examined using our novel paradigm.

\section{FURTHER POSSIBILITIES}

We constructed a setup where various behaviors of unconstrained flies can be recorded (Figure 1). We designed our conditioning paradigm in order to make it comparable to that commonly used in olfactory learning in many respects: the conditioning protocol, the appetitive US (sucrose) and its presentation with filter paper (Schwaerzel et al., 2003). Thus, appetitive memories of different sensory modalities can be directly compared. In several cases including ours, the same US application seems to be more effective on olfactory memory than visual memory (Hori et al., 2006; Krashes and Waddell, 2008; Colomb et al., 2009) (Figure 8).

The application of an LCD monitor allows generating a variety of visual stimuli. This opens a possibility for studying visual perception beyond associative memory per se. For example, the setup might be useful for studying color discrimination (intensity-independent spectral discrimination of light). Indeed, several seminal reports exploited visual associative learning to show the existence of such color vision in Drosophila and its psychophysical characteristics (Menne and Spatz, 1977; Bicker and Reichert, 1978; Hernández de Salomon and Spatz, 1983). Combined with a wide range of genetic techniques and resources, our setup may serve as a model to study the molecular and cellular mechanisms of insect vision.

\section{ACKNOWLEDGMENTS}

We thank A. Gruschka, B. Mühlbauer, and I. Siwanowicz for technical assistance; F. Banci, H. Kaderschabek, K. Öchsner, and H. Wintersberger for constructing custom parts of the setup; A. Borst, and T. Gollisch for experimental devices; Y. Aso, B. Gerber, M. Heisenberg, S. Knapek, R. Wolf, and S. Yamaguchi for discussion and/ or critical reading of the manuscript. This work was supported by the Emmy-Noether Program from Deutsche Forschungsgemeinschaft (H. Tanimoto), a PhD fellowship from Boehringer Ingelheim Fonds (C. Schnaitmann), and the Max-Planck-Gesellschaft. 


\section{REFERENCES}

Acevedo, S. F., Froudarakis, E. I., Kanellopoulos, A., and Skoulakis, E. M. (2007). Protection from premature habituation requires functional mushroom bodies in Drosophila. Learn. Mem. 14, 376-384.

Bicker, G., and Reichert, H. (1978). Visual learning in a photoreceptor degeneration mutant of Drosophila melanogaster. J. Comp. Physiol. A 127, 29-38.

Brembs, B., Christiansen, F., Pfluger, H. J., and Duch, C. (2007). Flight initiation and maintenance deficits in flies with genetically altered biogenic amine levels. J. Neurosci. 27, 11122-11131.

Chabaud, M. A., Isabel, G., Kaiser, L., and Préat, T. (2009). Social facilitation of long-lasting memory retrieval in Drosophila. Curr. Biol. 19, 1654-1659.

Colomb, J., Kaiser, L., Chabaud, M. A., and Préat, T. (2009). Parametric and genetic analysis of Drosophila appetitive long-term memory and sugar motivation. Genes Brain Behav. 8, 407-415.

de Brito Sanchez, M. G., Chen, C., Li, J., Liu, F., Gauthier, M., and Giurfa, M. (2008). Behavioral studies on tarsal gustation in honeybees: sucrose responsiveness and sucrose-mediated olfactory conditioning. J. Comp. Physiol. A 194, 861-869.

Fukushi, T. (1976). Classical conditioning to visual stimuli in the housefly, Musca domestica. J. Insect Physiol. 22, 361-364.

Fukushi, T. (1985). Visual learning in walking blowflies, Lucilia cuprina. J. Comp. Physiol. A 157, 771-778.

Fukushi, T. (1989). Learning and discrimination of coloured papers in the walking blowfly, Lucilia cuprina. J. Comp. Physiol. A 166, 57-64.

Gerber, B., and Hendel, T. (2006). Outcome expectations drive learned behaviour in larval Drosophila. Proc. Biol. Sci. 273, 2965-2968.

Gerber, B., Scherer, S., Neuser, K., Michels, B., Hendel, T., Stocker, R. F., and Heisenberg, M. (2004). Visual learning in individually assayed Drosophila larvae. J. Exp. Biol. 207, 179-188.

Giurfa, M., Fabre, E., Flaven-Pouchon, J., Groll, H., Oberwallner, B., Vergoz, V., Roussel, E., and Sandoz, J. C. (2009). Olfactory conditioning of the sting extension reflex in honeybees: memory dependence on trial number, interstimulus interval, intertrial interval, and protein synthesis. Learn. Mem. 16, 761-765.

Heisenberg, M. (1989). Genetic approach to learning and memory (mnemogenetics) in Drosophila melanogaster. In Fundamentals of Memory Formation: Neural plasticity and Brain Function,
H. Rahmann, ed. (Stuttgart, G. Fischer).

Heisenberg, M., Wolf, R., and Brembs, B. (2001). Flexibility in a single behavioral variable of Drosophila. Learn. Mem. 8, 1-10.

Hernández de Salomon, C., and Spatz, H. C. (1983). Colour vision in Drosophila melanogaster: wavelength discrimination. J. Comp. Physiol. A 150, 31-37.

Honjo, K., and Furukubo-Tokunaga, K. (2009). Distinctive neuronal networks and biochemical pathways for appetitive and aversive memory in Drosophila larvae. J. Neurosci. 29, 852-862.

Hori, S., Takeuchi, H., Arikawa, K., Kinoshita, M., Ichikawa, N., Sasaki, M., and Kubo, T. (2006). Associative visual learning, color discrimination, and chromatic adaptation in the harnessed honeybee Apis mellifera L. J. Comp. Physiol. A 192, 691-700.

Joseph, R. M., Devineni, A. V., King, I. F., and Heberlein, U. (2009). Oviposition preference for and positional avoidance of acetic acid provide a model for competing behavioral drives in Drosophila.Proc. Natl. Acad. Sci. U.S.A. 106, 11352-11357.

Kim, Y. C., Lee, H. G., and Han, K. A. (2007a). Classical reward conditioning in Drosophila melanogaster. Genes Brain Behav. 6, 201-207.

Kim, Y. C., Lee, H. G., and Han, K. A. (2007b). D1 dopamine receptor dDA1 is required in the mushroom body neurons for aversive and appetitive learning in Drosophila. J. Neurosci. 27, 7640-7647.

Krashes, M. J., and Waddell, S. (2008). Rapid consolidation to a radish and protein synthesis-dependent longterm memory after single-session appetitive olfactory conditioning in Drosophila. J. Neurosci. 28, 3103-3113.

Le Bourg, E., and Buecher, C. (2002). Learned suppression of photopositive tendencies in Drosophila melanogaster. Anim. Learn. Behav. 30, 330-341.

Lepot, F., and Médioni, J. (1986). Successive visual discrimination learning and memorisation in the dunce2 mutant of Drosophila melanogaster. In Genetic Approaches to Behaviour, J. Médioni and G. Vaysse, eds (Toulouse, Privat).

Liu, G., Seiler, H., Wen, A., Zars, T., Ito, K., Wolf, R., Heisenberg, M., and Liu, L. (2006). Distinct memory traces for two visual features in the Drosophila brain. Nature 439, 551-556.

McGuire, S. E., Deshazer, M., and Davis, R. L. (2005). Thirty years of olfactory learning and memory research in Drosophila melanogaster. Prog. Neurobiol. 76, 328-347.

Menne, D., and Spatz,H.C. (1977). Colour vision in Drosophila melanogaster. J. Comp. Physiol. A 114, 301-312.
Mery, F., and Kawecki, T. J. (2005). A cost of long-term memory in Drosophila. Science 308, 1148.

Mizunami, M., Unoki, S., Mori, Y., Hirashima, D., Hatano, A., and Matsumoto, Y. (2009). Roles of octopaminergic and dopaminergic neurons in appetitive and aversive memory recall in an insect. BMC Biol. 7, 46 .

Nakatani, Y., Matsumoto, Y., Mori, Y., Hirashima, D., Nishino, H., Arikawa, K., and Mizunami, M. (2009). Why the carrot is more effective than the stick: different dynamics of punishment memory and reward memory and its possible biological basis. Neurobiol. Learn. Mem. 92, 370-380.

Pitman, J. L., DasGupta, S., Krashes, M. J., Leung, B., Perrat, P.N., and Waddell, S. (2009). There are many ways to train a fly. Fly (Austin) 3, 3-9.

Quinn, W. G., and Dudai, Y. (1976). Memory phases in Drosophila. Nature 262, 576-577.

Quinn, W. G., Harris, W. A., and Benzer S. (1974). Conditioned behavior in Drosophila melanogaster. Proc. Natl. Acad. Sci. U.S.A. 71, 708-712.

Rescorla, R. A. (1988). Behavioral studies of Pavlovian conditioning. Annu. Rev. Neurosci. 11, 329-352.

Roussel, E., Carcaud, J., Sandoz, J. C. and Giurfa, M. (2009). Reappraising social insect behavior through aversive responsiveness and learning. PLoS ONE 4, e4197. doi: 10.1371/journal pone.0004197.

Scherer, S., Stocker, R. F., and Gerber, B. (2003). Olfactory learning in individually assayed Drosophila larvae. Learn. Mem. 10, 217-225.

Schipanski, A., Yarali, A., Niewalda, T., and Gerber, B. (2008). Behavioral analyses of sugar processing in choice, feeding, and learning in larval Drosophila. Chem. Senses 33, 563-573.

Schwaerzel, M., Monastirioti, M., Scholz, H., Friggi-Grelin, F., Birman, S., and Heisenberg, M. (2003). Dopamine and octopamine differentiate between aversive and appetitive olfactory memories in Drosophila. J. Neurosci. 23, 10495-10502.

Selcho, M., Pauls, D., Han, K. A., Stocker, R. F., and Thum, A. S. (2009). The role of dopamine in Drosophila larval classical olfactory conditioning. PLoS ONE 4, e5897. doi: 10.1371/journal. pone.0005897.

Shiraiwa, T., and Carlson, J. R.. (2007) Proboscis Extension Response (PER) Assay in Drosophila. J. Vis. Exp. 3. doi: 10.3791/193 (http://www.jove.com/ index/details.stp?id=193).

Spatz, H. C., Emanns, A., and Reichert, H. (1974). Associative learning of Drosophila melanogaster. Nature 248, 359-361.

Tempel, B. L., Bonini, N., Dawson, D. R., and Quinn, W. G. (1983). Reward learning in normal and mutant Drosophila. Proc Natl. Acad. Sci. U.S.A. 80, 1482-1486.

Thum, A. S., Jenett, A., Ito, K., Heisenberg, M., and Tanimoto, H. (2007). Multiple memory traces for olfactory reward learning in Drosophila. J. Neurosci. 27 11132-11138.

Tully, T., and Quinn, W.G. (1985). Classical conditioning and retention in normal and mutant Drosophila melanogaster. J. Comp. Physiol. A 157, 263-277.

Unoki, S., Matsumoto, Y., and Mizunami, M. (2005). Participation of octopaminergic reward system and dopaminergic punishment system in insect olfactory learning revealed by pharmacological study. Eur. J. Neurosci. 22, 1409-1416.

Unoki, S., Matsumoto, Y., and Mizunami, M. (2006). Roles of octopaminergic and dopaminergic neurons in mediating reward and punishment signals in insect visual learning. Eur. J. Neurosci. 24, 2031-2038.

van Swinderen, B., McCartney, A., Kauffman, S., Flores, K., Agrawal, K., Wagner, J., and Paulk, A. (2009). Shared visual attention and memory systems in the Drosophila brain. PLoS ONE 4, e5989. doi: 10.1371/journal. pone.0005989.

Vergoz, V., Roussel, E., Sandoz, J. C., and Giurfa, M. (2007). Aversive learning in honeybees revealed by the olfactory conditioning of the sting extension reflex. PLoS ONE 2, e288. doi: 10.1371/journal.pone.0000288.

Wolf, R., and Heisenberg, M. (1991). Basic organization of operant behavior as revealed in Drosophila flight orientation. J. Comp. Physiol. A 169, 699-705.

Wright, G. A., Mustard, J. A., Kottcamp, S. M., and Smith, B. H. (2007). Olfactory memory formation and the influence of reward pathway during appetitive learning by honey bees. J. Exp. Biol. 210, 4024-4033.

Conflict of Interest Statement: The authors declare that the research was conducted in the absence of any commercial or financial relationships that could be construed as a potential conflict of interest.

Received: 01 September 2009; paper pending published:07 November 2009; accepted: 08 February 2010; published online: 09 March 2010.

Citation: Schnaitmann C, Vogt K, Triphan $T$ and Tanimoto $H$ (2010) Appetitive and aversive visual learning in freely moving Drosophila. Front. Behav. Neurosci. 4:10. doi: 10.3389/fnbeh.2010.00010

Copyright (c) 2010 Schnaitmann, Vogt, Triphan and Tanimoto. This is an openaccess article subject to an exclusive license agreement between the authors and the Frontiers Research Foundation, which permits unrestricted use, distribution, and reproduction in any medium, provided the original authors and source are credited. 